\title{
Instruments and Data Logger for Measuring Electrical Parameters: Indonesian Market Review and Research Direction
}

\author{
A. S. Wibawa ${ }^{1}$, I. N. S. Kumara ${ }^{2}$, and I. W. Sukerayasa ${ }^{3}$ \\ ${ }^{1,2,3}$ Department Electrical Engineering \\ Faculty of Engineering at Udayana University \\ Bukit Jimbaran, Bali Indonesia 80361 \\ satya.kumara@unud.ac.id
}

\begin{abstract}
Electrical parameters such as voltage, current, power, energy, frequency, and power factor are parameters needed in the operation of an electrical system and machinery. The measurement results provide an overview of the system performance and indicator of abnormal or faulty operations. Nowadays, the electrical parameter instruments grow rapidly with a wide range of features and capability, started from analog instruments to digital measurement with data logging features. This paper reviews the availability of electrical parameter instruments and data logger systems in Indonesia. The review includes technical specifications of instruments, manufacturers, vendors, and data logging features. The data were obtained from vendor publications, manufacturers, scientific publications, and e-commerce websites in Indonesia. The information regarding the availability of the instruments and data logger systems will provide a quick reference in choosing instruments for a certain application to suit the purpose of the measurement and also the cost of the device. Moreover, the information also can provide benchmarks for research and development of low-cost multichannel data logging systems with additional features such as remotely accessible through the implementation of the internet of things.
\end{abstract}

Index Terms - Measuring instruments, electrical parameters, data logger, monitoring, voltage, current, power, energy, IOT

\section{INTRODUCTION}

Electrical energy plays an important role in the life of modern society. Ineffective and inefficient use of electricity can decrease non-renewable energy sources that are used in electricity generation. In utilizing electrical energy it is necessary to measure various electrical parameters in order to be able to maintain the quality of electric power itself. There are common electrical parameters, such as voltage, current, power, frequency, power factor, etc. Measuring the value of the electricity parameter is crucial to evaluate the performance of an electric machine or electronic system. Other than that, electricity parameters become an indicator of electrical system operation or disruptions [1]. Monitoring the electrical parameter is the most important thing to do in order to support the accuracy and speed of decision making when a problem occurs. The results of monitoring are present an overview of the electric power system performance.

Nowadays, the electrical parameter measurement instruments that have been developed have various types, started from the results of analog data using needles and readings using scales to digital readings. Even in one measuring instrument is capable of measuring various electrical parameters both AC and DC, such as AC and
DC voltage, AC and DC current, and the resistance or the instrument is known as a multimeter.

There are two measuring systems; they are analog systems, and digital systems. Electrical parameter measuring instruments with analog systems are used many interconnected mechanical components [2]. The most important part of an analog system is the electrical part which consisting of permanent magnets, resistors, swivel coils, while the mechanical part consists of a needle, scale, and needle regulating screw [2]. Meanwhile, the digital systems have analog data to measured but also have an analog to digital conversion (ADC) to convert analog input signals into digital that is processed with a microcontroller or microprocessor.

Measuring instrument must have several criteria, including the accuracy which is the proximity of the reading of the measuring instrument to the actual value, the precision that means the measurement results or degrees to distinguish one measurement from the other, sensitivity which means the ratio of the output signal or the response of the measuring instrument to changes input or measured variable. Then there is the resolution which is the smallest change from the measurement value that can be responded by the measuring instrument, as well as the error which is the number of deviations from the true value of the measured variable [2].

The development of measuring parameters lately has been equipped with data recording devices or data 
loggers. The data logger is an electronic device that can do data recording and data storage automatically from time to time both integrated with sensors and instruments inside as well as external sensors and instruments [3]. Electrical parameter measuring instruments equipped with data loggers is more expensive than measuring instruments without data loggers. The instruments that are equipped with a data logger can reach prices up to a hundred million rupiah depends on input channels number of instruments.

This paper will review the existence of electrical parameter measurement instruments and data logger systems in Indonesia. The aspects of the review include technical specifications, standardization/ certification/ labels, vendors, and manufacturers of data logger tools and systems. The data were obtained from vendor publications, manufacturers, scientific publications, certification/ standardization institutions, and ecommerce websites in Indonesia. Hopefully, the information on the existence of electrical parameter measurement instruments and data logger systems will help people to get quick access to references in choosing instruments for application purposes, and also it can be used as basic development research of low-cost multichannel data logging systems.

\section{STANDARD/LABELING ELECTRONIC EQUIPMENT}

Electrical units of the measurement system are used to express standard electrical units in order to make people easy to understand. There are two unit systems that can be used; they are the international system (SI) and the imperial system (Britain) that applies in Europe [4]. Since the 1960s the international unit system (SI) has been used as an international standard unit [2]. The SI unit contains a list of quantities and symbols that are used in the fields of electricity and magnetism worldwide.

TABLE I

ELECTRICAL PARAMETERS AND UNIT SI

\begin{tabular}{|c|c|c|}
\hline Parameters & Unit & Symbol \\
\hline Current (I) & Amper & A \\
\hline Voltage (V) & Volt & $\mathrm{V}$ \\
\hline Electromotive force $(\epsilon)$ & Volt & $\mathrm{V}$ \\
\hline Resistance (R) & ohm & $\Omega$ \\
\hline Electric Charger (Q) & coulomb & $\mathrm{C}$ \\
\hline Capacitance $(\mathrm{C})$ & Farad & $\mathrm{F}$ \\
\hline Electric Power $(\mathrm{P})$ & Watt & $\mathrm{W}$ \\
\hline Frequency $(\mathrm{F})$ & Herts & $\mathrm{Hz}$ \\
\hline Conductance $(\mathrm{G})$ & Siemens & $\mathrm{S}$ \\
\hline Magnetic Flux $(\Phi)$ & Weber & $\mathrm{Wb}$ \\
\hline Flux Density (B) & Tesla & $\mathrm{T}$ \\
\hline Inductance $(\mathrm{L})$ & Henry & $\mathrm{H}$ \\
\hline Energy $(\mathrm{E})$ & Joule & $\mathrm{J}$ \\
\hline
\end{tabular}

The certification of electronic products is used to guarantee the quality of the product is qualified in international standards, ensure user safety, and it has legal requirements in the countries where the product is being produced.

The standard various types that were adapted by the country where the product got its copyright. For electronic equipment, the certification products that are commonly used called CE, FCC, RoHS, UL, and WEEE.
FCC and UL are certifications used in the United States and the rest are used in Europe.

\section{A. Conformité Européenne (CE)}

The CE label is used to guarantee the health and safety of the users of products manufactured or sold in the European Economic Area (EEA) [36]. This EEP region includes 28 European Union (EU) countries, Iceland, Norway, Liechtenstein, Turkey, and Switzerland. This CE label must conform to EU regulations for electronic goods [37]

Electromagnetic Compatibility Directive (EMC) is an instruction to fulfill permitted electromagnetic emission levels. EMC regulates product durability in resisting electromagnetic emissions.

Low Voltage Directive (LVD) is a provision to ensure all products that use voltage levels from 50 to $1000 \mathrm{VAC}$ or 75 to 1500 VDC must guarantee safety when it used.

Radio Equipment Directive (RED) is a provision that regulates if the product is manufactured using wireless transmitters or communication equipment that uses radio waves. This is including setting frequency allocations and EMC standards.

\section{B. Restriction of Hazardous Substances (RoHS)}

RoHS is a directive that limits 10 hazardous materials in electronic and electrical products. RoHS prohibits the circulation of electronic devices that have heavy metal concentrations [38].

TABLE II

STANDARD CONCENTRATION OF HAZARDOUS MATERIAL ROHS

\begin{tabular}{|c|l|c|c|}
\hline \multirow{2}{*}{ No } & \multicolumn{2}{|c|}{ Hazardous Material } & \multicolumn{2}{c|}{ Concentration } \\
\cline { 3 - 4 } & & $\mathrm{ppm}$ & $\%$ \\
\hline 1 & Lead $(\mathrm{Pb})$ & $<1000$ & 0,1 \\
\hline 2 & Mercury $(\mathrm{Hg})$ & $<1000$ & 0,1 \\
\hline 3 & Cadamium $(\mathrm{Cd})$ & $<100$ & 0,001 \\
\hline 4 & Hexavalent Chromium (CrVI) & $<1000$ & 0,1 \\
\hline 5 & Polybrominated Biphenyls (PBB) & $<1000$ & 0,1 \\
\hline 6 & Polybrominated Diphenyl Ethers (PBDE) & $<1000$ & 0,1 \\
\hline 7 & Bis(2-Ethylhexyl) phthalate (DEHP) & $<1000$ & 0,1 \\
\hline 8 & Benzyl butyl phthalate (BBP) & $<1000$ & 0,1 \\
\hline 9 & Dibutyl phthalate (DBP) & $<1000$ & 0,1 \\
\hline 10 & Diisobutyl phthalate (DIBP) & $<1000$ & 0,1 \\
\hline
\end{tabular}

\section{Waste Electrical and Electronic Equipment (WEEE)}

WEEE is an instruction that regulates electronic and electrical manufacturers to develop products that can be recycled and have little electronic waste [37].

\section{D.Underwriters Laboratories (UL)}

UL standards are issued by Underwriters Laboratories. This standard is not obligatory like CE but will help to ensure that products launched are safe to use. This standard is used to assess component, material, system and performance tests that focus on safety standards and are certified Occupational Safety and Health Administration (OSHA) [37].

\section{E. Federal Communications Commission (FCC)}

FCC is a standard required for all products that emit electromagnetic or radio frequency (RF) signals in the frequency range of $9 \mathrm{kHz}$ or more. This standard serves to minimize the potential for equipment that emits radio frequencies to interfere with other devices [37]. 


\section{F. Indonesian National Standard (SNI)}

SNI is the only standard that applies nationally in Indonesia. SNI is determined and formulated by a technical committee established by the National Standardization Agency (BSN) [39]. There are around 400 SNIs related to electricity and electronics that have been issued by BSN [40]. Some SNI is related to data logger measurement tools can be shown in Table 3 .

TABLE III

INDONESIAN STANDARDS FOR ELECTRICAL MEASUREMENTS

\begin{tabular}{|c|c|c|}
\hline No & Standards & Scope \\
\hline 1 & $\begin{array}{l}\text { SNI IEC 61000- } \\
\text { 4-6:2014 }\end{array}$ & $\begin{array}{l}\text { Testing and measurement techniques - } \\
\text { Immunity to conduction disturbances induced } \\
\text { by radiofrequency fields (IEC } 61000-4-6 \text { ed } \\
\text { 3.0: } 2008 \text {, IDT) }\end{array}$ \\
\hline 2 & $\begin{array}{l}\text { SNI IEC 61000- } \\
4-3: 2014\end{array}$ & $\begin{array}{l}\text { Testing and measurement techniques - } \\
\text { Electromagnetic field immunity test, radiated } \\
\text { radio frequency (IEC } 61000-4-3 \text { ed } 3.2 \\
\text { Consolidation with am } 1 \& 2 ; 2010 \text {, IDT) }\end{array}$ \\
\hline 3 & $\begin{array}{l}\text { SNI IEC 60051- } \\
1: 2009\end{array}$ & $\begin{array}{l}\text { Direct measuring analog electrical measuring } \\
\text { instruments and accessories - Part } 1 \text { : General } \\
\text { definitions and requirements along with all } \\
\text { parts. }\end{array}$ \\
\hline 4 & $\begin{array}{c}\text { SNI 04- } \\
6267.302-2001\end{array}$ & $\begin{array}{l}\text { Electrical engineering terminology - Chapter } \\
\text { 302: Electrical measuring instruments. }\end{array}$ \\
\hline 5 & $\begin{array}{c}\text { SNI 04- } \\
6267.303-2002\end{array}$ & $\begin{array}{l}\text { Electrical engineering terminology Chapter: } \\
303 \text { Electronic measuring instruments. }\end{array}$ \\
\hline 6 & $\begin{array}{c}\text { SNI IEC } \\
61786: 2011\end{array}$ & $\begin{array}{l}\text { Measurement of instrument fields and low- } \\
\text { frequency electricity exposed to humans. } \\
\text { Specific requirements for instruments and } \\
\text { measurement guidelines. }\end{array}$ \\
\hline 7 & $\begin{array}{l}\text { SNI IEC } 60051- \\
\quad 3: 2009\end{array}$ & $\begin{array}{l}\text { Direct instructions for analog electrical } \\
\text { measuring equipment and accessories - Part } 3 \text { : } \\
\text { special requirements for watt meters and } \\
\text { varmeters. }\end{array}$ \\
\hline 8 & $\begin{array}{l}\text { SNI IEC 62056- } \\
21: 2009\end{array}$ & $\begin{array}{l}\text { Data exchange for meter reading, load control } \\
\text { and tariffs Section 21: Direct local data } \\
\text { exchange. }\end{array}$ \\
\hline 9 & $\begin{array}{l}\text { SNI 04-6530- } \\
2001\end{array}$ & $\begin{array}{l}\text { Measurement of the power frequency electric } \\
\text { field }\end{array}$ \\
\hline 10 & $\begin{array}{l}\text { SNI 04-3875- } \\
\quad 1995\end{array}$ & $\begin{array}{l}\text { Special requirements for ohmmeters } \\
\text { (Impedance Meters) and Analog Pointing } \\
\text { conductance Meters and their Accessories. }\end{array}$ \\
\hline
\end{tabular}

\section{G.Regulatory Compliance Mark (RCM)}

RCM is a label for importers and manufacturers from Australia and New Zealand. This RCM follows the standards derived from, Electrical Equipment Safety System (EESS), Electro Magnetic Compatibility (EMC), Electromagnetic Radiation (EMR) and Australian telecommunications requirements [41].

\section{H.Technischer Überwachungsverein (TÜV Rheinland)}

$T \ddot{U} V$ Rheinland is a certification of safety and quality standards of low voltage equipment, and an electric power distribution system equipment. TÜV Rheinland has several national certification bodies throughout the world and testing laboratories including $T \ddot{U} V$ Rheinland Inter Cert Hungary, a national member body in the IECEE CB scheme that is recognized worldwide [42].
TABLE IV

STANDARD OR LABELING FOR FI FCTRONIC EQUIPMENT

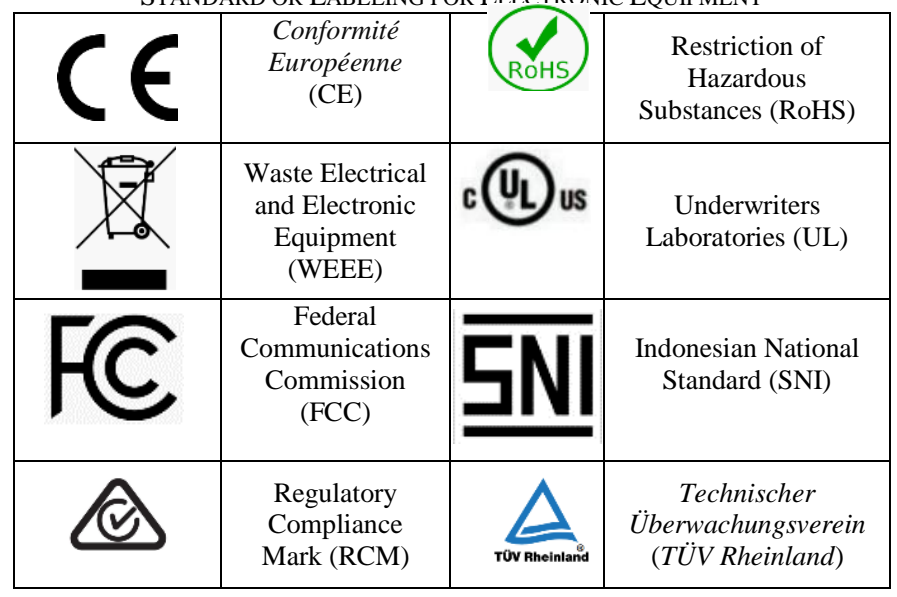

\section{MATERIAL AND METHODS}

The data that is used were technical specifications, manufacturers, vendors, standardization/labels and data logger systems. Data were obtained from technical datasheet publications from manufacturers, information from vendors, certification bodies in the country and internationally, scientific publications, and e-commerce websites in Indonesia.

Data processing were begun by gathering all information about the specifications and technical data of electrical parameter measurement instruments and data logger systems that sold in the national market, then the data were grouped into two categories, they are electrical parameter measurement instruments with a data logger system and without a data logger system. Data that has been grouped were sorted again from the lowest to the most expensive prices and then an analysis of what factors affect the price difference between the items in the same group was carried out. The results of this analysis were expected to be one of the quick references for people or agencies who need it and to be the basis for research into the development of low-cost multichannel data logging systems. Figure 1 is a general schematic of the research methodology.

\section{RESUlTS AND DisCUSSION}

\section{A.Electrical Parameters Measurement}

An electrical measuring instrument was a device used to measure electrical quantities. There are various types of electrical measuring instruments that have been circulating in the market which had different specifications. In Table 5 the electrical measuring instruments were described which can be used to measure electrical parameters but those instruments were not equipped with a data logger system yet.

Those instruments were used to monitor electrical parameters, most of them had a compact size so they were easy to carry everywhere. They also were equipped with a power supply with a battery so it made easier to use. The electrical parameter measurement instruments that were not equipped with a data logger system was cheaper than the instruments with data logger system. 


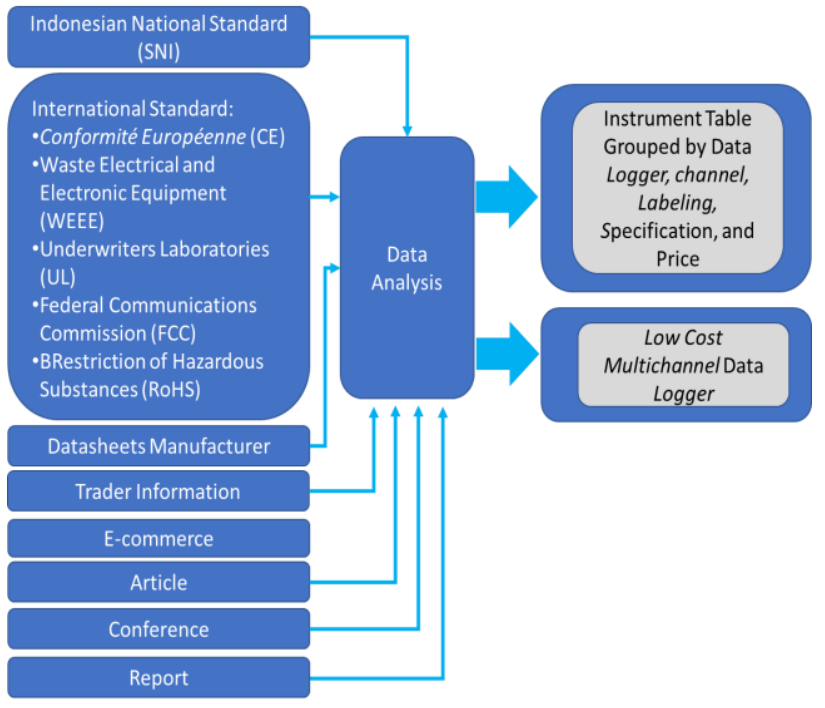

Fig. 1. General schematic research

At the time of this research, the exchange rate of USD to Indonesian Rupiah was 1 USD, equivalent to Rp. 13,986.

TABLE V

ELECTRICAL PARAMETERS MEASUREMENT INSTRUMENT

\begin{tabular}{|c|c|c|c|}
\hline No & \multicolumn{2}{|c|}{ Name of Measuring In strument } & Specification \\
\hline \multirow{4}{*}{1} & Product & Deek-Robot BL-02 & \multirow{4}{*}{$\begin{array}{l}\text { - Current: } 0-5 \mathrm{~A} \\
\text { - Voltage: DC 0-50V } \\
\text { - Power: } 0-250 \mathrm{~W} \\
\text { - T emperature: }-10-60 \mathrm{C} \\
\text { - accuracy }+-1 \% \\
\text { - Dimen sion : } 79.5 * 43 * 3 \mathrm{~mm}\end{array}$} \\
\hline & Description & $\begin{array}{l}\text { Digital Voltage } \\
\text { Current Power Meter }\end{array}$ & \\
\hline & Certification & - & \\
\hline & Price (Rp) & 77.000 & \\
\hline \multirow{4}{*}{2} & Product & KWE-PMDI-EU & \multirow{4}{*}{$\begin{array}{l}\text { - Voltage : } 230 \mathrm{~V}-250 \mathrm{~V} \text { AC } \\
\text { - Current: } \max 16 \mathrm{~A} \\
\text { - Power: } 0 \mathrm{~W}-3680 \mathrm{~W} \\
\text { - Frequency: } 50 \mathrm{~Hz} \\
\text { - Can display values } \mathrm{kWh} \text { used } \\
\text { - Dimen sion }: 5.5 \times 7.0 \times 3.5 \mathrm{~cm}\end{array}$} \\
\hline & Description & $\begin{array}{l}\text { Power Analyzer } \\
\text { Digital Watt Voltage } \\
\text { Ampere Meter }\end{array}$ & \\
\hline & Certification & CE, WEEE & \\
\hline & Price (Rp) & 135.000 & \\
\hline
\end{tabular}

Based on Table 5. It can be seen that the price of measuring instrument varies from $\mathrm{Rp} 77.000$ to $\mathrm{Rp}$ 10.500.000 depends on several factors, such as the range or limit of measurement of electrical parameters that can be measured by the device, the type of electrical parameters that can be measured, the type of AC / DC system measured, the ability of the device to be interconnected wirelessly, the display of information that can be displayed on the display LCD. Some instruments in table 2 that were not equipped with a label from the country where the equipment was being produced were TCXRE AC 80-300 V, JUNTEK DC VAT-1100, DeekRobot BL-02.
TABLE V CONTINUED

ELECTRICAL PARAMETERS MEASUREMENT INSTRUMENT

\begin{tabular}{|c|c|c|c|}
\hline No & \multicolumn{2}{|c|}{ Name of Measuring In strument } & Specification \\
\hline \multirow{4}{*}{3} & Product & PZEM-051 100V 100A & \multirow{4}{*}{$\begin{array}{l}- \text { Voltage : } 6.5-100 \mathrm{~V} \\
\text { - Current: } 0-100 \mathrm{~A} \cdot \text { Accuracy } 1 \% \\
\text { - Power: } 0-10 \mathrm{~kW} \\
\text { - Energy: } 0-9999 \mathrm{kWh} \\
\text { - Dimension: } 89.6 \times 49.6 \mathrm{x} 24.4 \mathrm{~mm}\end{array}$} \\
\hline & Description & $\begin{array}{l}\text { DC } 4 \text { in } 1 \text { Volt, Amper, } \\
\text { Watt. Energy meter }\end{array}$ & \\
\hline & Certification & CE, FCC, WEEE & \\
\hline & Price (Rp) & 200.000 & \\
\hline \multirow{4}{*}{4} & Product & JPN & \multirow{4}{*}{$\begin{array}{l}\text { - Dimension : } 8,4 \times 5 \times 2 \mathrm{~cm} \\
\text { - Voltage: } 0-60 \mathrm{~V} \cdot \text { Current: } 0-100 \mathrm{~A} \\
\text { - Power: } 0-6554 \mathrm{~W} \\
\text { - Battery capacity:0-65Ah } \\
\text { - Energy: } 0-6554 \mathrm{Wh}\end{array}$} \\
\hline & Description & DC Watt Ampere Volt & \\
\hline & Certification & $\mathrm{CE}$ & \\
\hline & Price (Rp) & 230.000 & \\
\hline & Product & GT Power $150 \mathrm{~A}$ & \multirow{4}{*}{ 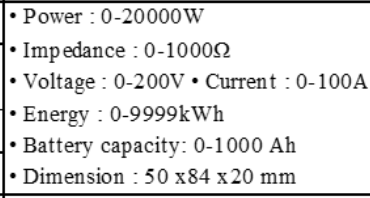 } \\
\hline 5 & Description & $\begin{array}{l}\text { High Precision Power } \\
\text { An alyzer \& Watt }\end{array}$ & \\
\hline & Certification & FCC, CE, WEEE & \\
\hline & Price (Rp) & 235.000 & \\
\hline \multirow{4}{*}{6} & Product & PZEM-013 & \multirow{4}{*}{$\begin{array}{l}\text { - Power: } 0-20000 \mathrm{~W} \\
\text { - Impedance : } 0-1000 \Omega \\
\text { - Voltage: } 0-200 \mathrm{~V} \\
\text { - Current : } 0-100 \mathrm{~A} \\
\text { - Energy : } 0-9999 \mathrm{kWh} \\
\text { - Battery capacity: } 0-1000 \mathrm{Ah} \\
\text { - Dimensi } 50 \times 8 \times 20 \mathrm{~mm}\end{array}$} \\
\hline & Description & $\begin{array}{l}\text { Digital Current, } \\
\text { Voltage, Solar Power } \\
\text { Meter Multimeter }\end{array}$ & \\
\hline & Certification & CE, FCC, WEEE & \\
\hline & Price (Rp) & 282.550 & \\
\hline & Product & TCXRE AC $80-300 \mathrm{~V}$ & \multirow{4}{*}{$\begin{array}{l}- \text { AC Measurement } 50 \mathrm{~Hz} \\
\text { - Voltage : } 80-300 \mathrm{~V} \cdot \text { A ccuracy } 1 \% \\
\text { - Current : 0-100 A } \\
\text { - Power : 0-30000 W } \\
\text { - Energy : 0-99999 kWh } \\
\text { - Dimensi : } 8 \times 4.2 \times 4.6 \mathrm{~cm}\end{array}$} \\
\hline 7 & Description & $\begin{array}{l}\text { LCD 100A Volt Watt } \\
\text { Power Meter Amper } \\
\text { Meter Volt Meter }\end{array}$ & \\
\hline & Certification & - & \\
\hline & Price (Rp) & 318.000 & \\
\hline \multirow{4}{*}{8} & Product & $\begin{array}{l}\text { JUNT EK DC VAT - } \\
1100\end{array}$ & \multirow{4}{*}{$\begin{array}{l}- \text { Voltage: } 0.01-100 \mathrm{~V} \\
\text { - Current: } 0.1-100 \mathrm{~A} \\
\text { - T emperature }-20^{\circ} \mathrm{C}-120^{\circ} \mathrm{C} \\
\text { - Energy: } 0-4000 \mathrm{KWH} \\
\text { - Wireless Channel Setting A-Z(26 } \\
\text { channels) }- \text { Power: } 0-200 \mathrm{KW} \\
\text { - Display: } 1.8^{\prime \prime} \text { T FT LCD Display } \\
\text { - Wireless: up to } 10 \text { meters } \\
\text { - Dimension : } 98 * 54 * 21 \mathrm{~mm}\end{array}$} \\
\hline & Description & $\begin{array}{l}\text { Wireless Digit al Bi- } \\
\text { direct ional Power } \\
\text { Meter Ammeter } \\
\text { Voltmeter Capacity } \\
\text { Coulomb Counter } \\
\end{array}$ & \\
\hline & Certification & - & \\
\hline & Price (Rp) & 517.000 & \\
\hline & Product & Ky oritsu $2117 \mathrm{R}$ & \multirow{4}{*}{$\begin{array}{l}\cdot \text { Measurement Method True RMS } \\
\text { - AC/ DC Current } 60 / 600 / 1000 \mathrm{~A} \\
\text { - AC/DC Voltage } 6 / 60 / 600 \mathrm{~V} \\
\text { - Resistance } 600.0 / 6.000 / 60.00 / 600.0 \\
\text { k ohm - Cable Max } 33 \mathrm{~mm} \\
\text { - Equipped with Buzzer }\end{array}$} \\
\hline 9 & Description & $\begin{array}{l}\text { Digital Clamp Meter } \\
1000 \mathrm{~A} \text { AC/DC }\end{array}$ & \\
\hline 9 & Certification & $\mathrm{CE}, \mathrm{RoHS}$ & \\
\hline & Price (Rp) & 825.000 & \\
\hline \multirow{4}{*}{10} & Product & Fluke 179 T rue-RMS & \multirow{4}{*}{$\begin{array}{l}\text { - Voltage DC/AC : Max } 1000 \mathrm{~V} \\
\text { - Current AC/DC : } 10 \mathrm{~A} \\
\text { - Resistance }: 50 \mathrm{M} \Omega \\
\text { - Capasitance }: 10.000 \mu \mathrm{F} \\
\text { - Frequency : Max } 100 \mathrm{kHz} \\
\text { - Temperature }:-400 \mathrm{C} \text { up to } 4000 \mathrm{C}\end{array}$} \\
\hline & Description & Digital Multimeter & \\
\hline & Certification & $\begin{array}{l}\text { CE, CSA, WEEE, } \\
\text { RCM, T UV,VDE }\end{array}$ & \\
\hline & Price (Rp) & 4.150 .000 & \\
\hline \multirow{4}{*}{11} & Product & Fluke 381 & - Current DC/AC: $\max 1000 \mathrm{~A}$ \\
\hline & Description & $\begin{array}{l}\text { Remote Disp lay T rue- } \\
\text { rms AC/DC Clamp } \\
\text { Meter with iFlex }{ }^{\mathrm{TM}} \\
\end{array}$ & $\begin{array}{l}\text { - Voltage } \mathrm{AC} / \mathrm{DC}: 600 \mathrm{~V} / 1000 \mathrm{~V} \\
\text { - Frequency : } 5,0-500 \mathrm{~V} \\
\text { - Resistance : } 600 \Omega-60 \mathrm{k} \Omega\end{array}$ \\
\hline & Certification & CE, CSA, WEEE, RCM & - The display can connected to the \\
\hline & Price (Rp) & 10.500 .000 & \\
\hline
\end{tabular}




\section{V.INSTRUMENTS WITH DATA LOGGER}

Data loggers had been widely used not only in the electronic field but in all systems related to technology [5]. the data logger was an electronic device that can collect and record data automatically from time to time. Parameter measurement equipment that was equipped with a data logger system was widely used in monitoring power systems, both used in substations, and power generation systems that utilize renewable energy, as well as in industry. With this data logger, it made the user easier monitoring the system, so it would be easier to get a picture of an electrical or electronic system condition.

Many of these tools were equipped with systems that can connect to the internet, so that simplify users to access their data remotely. Some measuring instruments that used data loggers were also equipped with a solidstate drive (SSD) memory, so that the access to data storage, reading data from memory can be more quickly and safely.

TABLE VI

INSTRUMENTS WITH DATA LOGGER

\begin{tabular}{|c|c|c|c|}
\hline No & \multicolumn{2}{|c|}{ Name of Measuring Instrument } & Specification \\
\hline \multirow{4}{*}{1} & Product & Hantek 365F & \multirow{4}{*}{$\begin{array}{l}\text { - Data logger single channel } \\
\text { - Measurement type True RMS } \\
\text { - Data logger current, voltage, resistance, capacitance } \\
\text { - That can produce a trend in real-time. } \\
\text { - That can be interconnected with iPad, Android, dan send the data via Bluetooth } \\
\text { - Voltage DC } 60 \mathrm{mV}-800 \mathrm{~V} \text {, Voltage AC: } 60 \mathrm{mV}-600 \mathrm{~V} \\
\text { - Current DC: } 60 \mathrm{~mA} \text { - } 10 \mathrm{~A} \text {, Current AC: } 60 \mathrm{~mA}-10 \mathrm{~A} \\
\text { - Resistance: } 600-60 \mathrm{M} \Omega \text { C Capacitance: } 40 \mathrm{nF}-400 \mathrm{~F} \cdot \text { Temperature: } 0-1000^{\circ} \mathrm{C}\end{array}$} \\
\hline & Description & $\begin{array}{l}\text { USB digital data logger } \\
\text { recorder multimeter voltage } \\
\text { and current }\end{array}$ & \\
\hline & Certification & CE, WEEE & \\
\hline & Price $(\mathrm{Rp})$ & 2.218 .000 & \\
\hline \multirow{4}{*}{2} & Product & $\begin{array}{l}\text { DL150 EXTECH EX0000266 } \\
\text { Inc. PPN }\end{array}$ & \multirow{4}{*}{$\begin{array}{l}\text { - Single Channel } \\
\text { - Measurement type True RMS } \\
\text { - Voltage: } 10 \mathrm{~V} \text { to } 600 \mathrm{~V} \\
\text { - Current: } 10 \text { A to } 200 \mathrm{~A} \\
\text { - Data save in software PC with USB and format spreadsheet } \\
\text { - LCD Display }\end{array}$} \\
\hline & Description & $\begin{array}{l}\text { AC Voltage and Current Data } \\
\text { Logger }\end{array}$ & \\
\hline & Certification & $\mathrm{CE}$ & \\
\hline & Price $(\mathrm{Rp})$ & 5.141 .000 & \\
\hline \multirow{4}{*}{3} & Product & Hioki LR5043-20 & \multirow{4}{*}{$\begin{array}{l}\text { - Single-channel }- \text { DC Measurement } \\
\text { - Max Voltage } 50 \mathrm{~V} \cdot \text { LCD display } \\
\text { - It can still record data even if the battery is removed in } 30 \text { seconds. } \\
\text { - Waterproof and dustproof (IP 54) } \\
\text { - There is a system back up data when the error/ low batteries happen }\end{array}$} \\
\hline & Description & Single Channel Voltage Logger & \\
\hline & Certification & CE, WEEE & \\
\hline & Price $(\mathrm{Rp})$ & 6.995 .000 & \\
\hline \multirow{4}{*}{4} & Product & Onset EG4115 Core & \multirow{4}{*}{$\begin{array}{l}\text { - } 15 \text { channel } \\
\text { - Using SSD memory } \\
\text { - Voltage AC 0- } 277 \text { V RMS AC } \\
\text { - Current: max } 6900 \mathrm{~A} \\
\text { - A web server that allows users to connect to the interface via the internet } \\
\text { - Data logger that stores parameters V, A, W, Wh, Hz, VA, VAr, THD, deg.t }\end{array}$} \\
\hline & Description & 15 Input Meter Data Logger & \\
\hline & Certification & CE, UL, FCC & \\
\hline & Price (Rp) & 13.335 .000 & \\
\hline \multirow{4}{*}{5} & Product & Extech 380803 & \multirow{4}{*}{$\begin{array}{l}\text { - True RMS, } 1 \text { phase } \\
\text { - This using to analyze AC/DC load } \\
\text { - Data logger that can save } 1,012 \text { data } \\
\text { - Data that can be measured are Active Power }(\mathrm{W}) \text {, Power factor, apparent power (VA), } \\
\text { Voltage, Frequency, Current } \\
\text { - Voltage } 200 \mathrm{~V} / 750 \mathrm{~V} \text {. C Current } 2 / 20 \mathrm{~A} \text { from terminal and } 2 / 15 \mathrm{~A} \text { from a socket } \\
\text { - Power } 200 / 2000 \mathrm{~W} \cdot \text { Power factor } 0.5-1,0 \cdot \text { Accuracy } \pm 0,5 \%\end{array}$} \\
\hline & Description & $\begin{array}{l}\text { True RMS Power } \\
\text { Analyzer/Appliance Tester and } \\
\text { Datalogger }\end{array}$ & \\
\hline & Certification & $\mathrm{UL}, \mathrm{CE}$ & \\
\hline & Price (Rp) & 15.500 .000 & \\
\hline \multirow{4}{*}{6} & Product & Onset T-VER-E50B2 & \multirow{4}{*}{$\begin{array}{l}\text { - True RMS, one or three-phase AC } \\
\text { - Measurement of AC voltage, battery capacity (Ah), Current (A), kilowatt-hours (kWh), kilo } \\
\text { watts (kW), Power Factor (PF), Reactive Volt-Amp, Volt-Reactive Amp Hours, Volt- } \\
\text { Amps (VA), Volts (V), Watt Hours (Wh) and Watts (W) } \\
\text { - Current: } 5 \text { - 32000 A } \\
\text { - UL Maximums: 600VL-L (347VL-N) • CE Maximums: 300VL-N (520V L-L) }\end{array}$} \\
\hline & Description & Electric Power Data logger & \\
\hline & Certification & UL, CE, RoHS & \\
\hline & Price $(\mathrm{Rp})$ & 15.655 .000 & \\
\hline \multirow{4}{*}{7} & Product & Graphtec GL240 & \multirow{4}{*}{$\begin{array}{l}\text { - } 10 \text { analog channels, } 4 \text { digital channels } \bullet 4 \text { alarm outputs } \\
\text { - 4GB Internal memory, SDHC card slot (max } 32 \mathrm{~GB}) 2 \text { slot } \\
\text { - data saved to CSV format dan GBD Binary } \\
\text { - Interconnection with a Web server to remote monitoring, and send data to e-mail } \\
\text { - Voltage } 20 \mathrm{mV}-100 \mathrm{~V} \cdot \text { Current } 4-20 \mathrm{~mA} \cdot \text { Thermocouple measurement K,J,E,T,R,S,B,N,W. }\end{array}$} \\
\hline & Description & 10 analog channels & \\
\hline & Certification & $\mathrm{CE}$ & \\
\hline & Price (Rp) & 22.907 .500 & \\
\hline
\end{tabular}




\begin{tabular}{|c|c|c|c|}
\hline No & & Instruments & Specification \\
\hline \multirow{4}{*}{8} & Product & Lutron DW-6095 & \multirow{4}{*}{$\begin{array}{l}\text { - True RMS } \\
\text { - Parameter AC, Power Factor, Harmonic, Crest Factor, Apparent, Reactive Power, THD } \\
\text { - Voltage } 600 \mathrm{~V} \cdot \text { Current : } 1200 \mathrm{~A} \\
\text { - Real-time logger } 2 \text { s to } 7200 \mathrm{~s} \\
\text { - Data transient like dip, swell dan outage } \text { P Power system analyser } \\
\text { - Show waveform, harmonics, Total Harmonic Distortion, phase diagram } \\
\text { - save the data in SD card to EXCEL software to analyze }\end{array}$} \\
\hline & Description & $\begin{array}{l}\text { Power analyzer real-time data } \\
\text { logger }\end{array}$ & \\
\hline & Certification & $\mathrm{CE}$ & \\
\hline & Price $(\mathrm{Rp})$ & 30.000 .000 & \\
\hline \multirow{4}{*}{9} & Product & AEMC DL-1080 & \multirow{4}{*}{$\begin{array}{l}\text { - } 8 \text { - } 16 \text { channel data logger } \\
\text { - able to measurement Thermocouple, Pt100, Pt1000, voltage, and current DC } \\
\text { - Using SD card to save the data up to } 16 \mathrm{~GB} \\
\text { - Voltage : } 20 \mathrm{mV}, 50 \mathrm{mV}, 60 \mathrm{mV},-20 \text { to }+20 \mathrm{mV}, 0 \text { to } 5 \mathrm{~V}, 0 \text { to } 10 \mathrm{~V} \\
\text { - Current: } 0 \text { to } 20 \mathrm{~mA}, 4 \text { to } 20 \mathrm{~mA} \\
\text { - Thermocouples J, K, T, E, N, R, S \& B } \\
\text { - RTD100 (PT100) \& RTD1000 (PT1000) }\end{array}$} \\
\hline & Description & Data Logger & \\
\hline & Certification & $\mathrm{CE}$ & \\
\hline & Price (Rp) & 40.500 .000 & \\
\hline \multirow{4}{*}{10} & Product & Hioki PW 3365-20 & \multirow{4}{*}{$\begin{array}{l}\text { - } 3 \text { channel Voltage and } 3 \text { channel Current (Multichannel) } \\
\text { - Measurement method True RMS } \\
\text { - AC } 3 \text { phase, } \cdot \text { Voltage : } 90 \mathrm{~V}-520 \mathrm{~V}, \cdot \text { Power : } 200 \mathrm{~W}-6.0000 \mathrm{MW} \\
\text { - } \text { - Curre data to SD card, LAN dan USB } \\
\text { - Data storage time is } 1 / 2 / 5 / 10 / 15 / 30 \text { seconds and } 1 / 2 / 5 / 10 / 30 / 60 \text { minutes }\end{array}$} \\
\hline & Description & Clamp Power Logger & \\
\hline & Certification & $\mathrm{CE}$ & \\
\hline & Price (Rp) & 41.300 .000 & \\
\hline \multirow{4}{*}{11} & Product & Fluke 2638A/05 120 & \multirow{4}{*}{$\begin{array}{l}\text { - Measurement method True RMS } \\
\text { - } 22 \text { channels; } 8 \text { digital } \mathrm{I} / \mathrm{O} \text { dan } 6 \text { alarm outputs (TTL) } \\
\text { - Measurement of ac V, dc V, ac I, dc I, Thermocouple, PRT }(2,3,4 \mathrm{w}) \text {, } \\
\text { thermistor, resistance }(2-4 \mathrm{w}) \cdot \text { Support using USB flash drive } \\
\text { - Voltage : max } 300 \mathrm{~V} \cdot \text { Resistance }: 100 \Omega \text { to } 100 \mathrm{M} \Omega \\
\text { - J dan k Thermocouple: }-200^{\circ} \mathrm{C} \text { to } 1,000^{\circ} \mathrm{C}, \cdot \mathrm{K} \text { Thermocouple: }-200^{\circ} \mathrm{C} \text { to } 1,000^{\circ} \mathrm{C} \\
\text { - RTD (PT100): }-200^{\circ} \mathrm{C}, \cdot \mathrm{T} \text { Thermocouple : }-270^{\circ} \mathrm{C} \text { to } 400^{\circ} \mathrm{C}\end{array}$} \\
\hline & Description & $\begin{array}{l}22 \text { Channel hydra series III data } \\
\text { loggers }\end{array}$ & \\
\hline & Certification & CE, CSA, WEEE & \\
\hline & Price (Rp) & 61.000 .000 & \\
\hline \multirow{4}{*}{12} & Product & Fluke $3540 \mathrm{FC}$ & \multirow{4}{*}{$\begin{array}{l}\text { - Multi Channel, } \bullet \text { Voltage: Max } 1000 \mathrm{~V} \text { RMS } \\
\text { - Current: Max } 6000 \mathrm{~A} \\
\text { - Data can be access with Wi-fi }- \text { Uploading data to cloud and mobile fluke application } \\
\text { - } 1 \text { or } 3 \text { phase } \\
\text { - Measure Voltage, current, frequency, power factor, active power, and reactive power } \\
\text { - Total harmonic distortion (THD) } \text { Show trends graphic and fluctuations }\end{array}$} \\
\hline & Description & $\begin{array}{l}\text { Three-Phase Power Monitor } \\
\text { and Condition Monitoring Kit }\end{array}$ & \\
\hline & Certification & CE, CSA, WEEE & \\
\hline & Price (Rp) & 74.500 .000 & \\
\hline \multirow{4}{*}{13} & Product & FLUKE 1735 & \multirow{4}{*}{$\begin{array}{l}\text { - } 3 \text { phase power system } \\
\text { - Voltage Wye: } 57 \mathrm{~V} \text { - } 480 \mathrm{~V} \text { AC RMS } \\
\text { - Voltage Delta: } 100 \mathrm{~V}-830 \mathrm{~V} \text { AC RMS } \\
\text { - Current: } 15 \mathrm{~A} / 150 \mathrm{~A} / 3000 \mathrm{~A} \text { RMS } \\
\text { - Recording active power, reactive power, apparent power, power factor, and harmonic } \\
\text { distortion. (kWh, kVAh, kVARh) }\end{array}$} \\
\hline & Description & Three-Phase Power Logger & \\
\hline & Certification & CE, CSA, WEEE & \\
\hline & Price (Rp) & 85.000 .000 & \\
\hline \multirow{4}{*}{14} & Product & Hioki LR8400-93 & \multirow{4}{*}{$\begin{array}{l}\text { - } 30 \text { channel data loggers } \\
\text { - Can be combined with Matlab } \cdot \text { Measurement of solar module parameters } \\
\text { - Current } 2000 \text { A RMS AC/DC with a clamp-on current sensor. } \\
\text { - Voltage } 1 \mathrm{~V} \text { f. s, } 1,000 \mathrm{~V} \mathrm{rms} \\
\text { - Measurement of Insolation, } 20 \mathrm{mV} \text { f.s., } 140 \mathrm{~kW} / \mathrm{m} 2 \\
\text { - Temperature measurement mode, } \mathrm{K} \text { thermocouple } 100{ }^{\circ} \mathrm{C} \text { f.s., } 1{ }^{\circ} \mathrm{C}\end{array}$} \\
\hline & Description & PV Power Verifier & \\
\hline & Certification & $\mathrm{CE}$ & \\
\hline & Price (Rp) & 106.000 .000 & \\
\hline \multirow{4}{*}{15} & Product & FLUKE 435-II & \multirow{4}{*}{$\begin{array}{l}\text { - Measurement method True RMS }- \text { Has a logger function, } 1 \text { data storage of max 15MB } \\
\text { - Used to measure AC output power and DC, V RMS, Arms, V peak, } \\
\text { A peak, V Crest Factor, A Crest Factor, Hz, Vrms } 1 / 2, \text { Arms } 1 / 2, \text { Watt, VA, VAR, power } \\
\text { factor Cos } \phi / \text { DPF, Inrush current } \\
\text { - Monitoring power quality parameter (Harmonics, Unbalance, Flicker, Transient) } \\
\text { - Voltage: } 1000 \mathrm{~V} \text { rms, } \cdot \text { Current: } 20 \mathrm{k} \text { Arms } \\
\text { - Calculate the lost energy and calculate it in nominal money }\end{array}$} \\
\hline & Description & $\begin{array}{l}\text { Three-Phase Power Quality and } \\
\text { Energy Analyzer }\end{array}$ & \\
\hline & Certification & UL, CE, CSA, WEEE & \\
\hline & Price (Rp) & 174.000 .000 & \\
\hline \multirow{4}{*}{16} & Product & Hioki MR8827 & \multirow{4}{*}{$\begin{array}{l}\text { - } 32 \text { analogue }+32 \text { logic channels + FFT } \\
\text { - Measurement method True RMS with optional PROBE like } 9322 \\
\text { - Monitoring for voltage, current, temperature, vibration, weight, } \\
\text { acceleration and rotation. } \\
\text { - Voltage } 200-500 \mathrm{~V} \text { optional sensor } \\
\text { - Current } 100 \mathrm{~A}-5000 \mathrm{~A} \text { depending on the clamp used } \\
\text { - }[16 \text { analogue input modules]: } 32 \text { analogue channels }+32 \text { logic channels } \\
\text { - }[14 \text { analogue input modules }+2 \text { logic input modules]: } 28 \text { analogue Channels }+64 \text { logic } \\
\text { - Voltage: } 5 \mathrm{mV} / \text { div to } 20 \mathrm{~V} / \text { div } \\
\text { - Memory (high-speed recording) SSD } 128 \mathrm{~GB} \text { to save } 100 \mathrm{MB} \text { data }\end{array}$} \\
\hline & Description & $\begin{array}{l}\text { Memory HiCorder } 32 \text { Analog } \\
\text { channel and } 32 \text { logic channel }\end{array}$ & \\
\hline & Certification & $\mathrm{CE}$ & \\
\hline & Price (Rp) & 214.500 .000 & \\
\hline \multirow{4}{*}{17} & Product & Hioki MR8740 & \multirow{4}{*}{$\begin{array}{l}\text { - Multichannel up to } 54 \text { channels } \\
\text { - FFT analysis } \\
\text { - Measurement method True RMS with optional PROBE like } 9322 \\
\text { - Voltage200 - 500 V optional sensor } \\
\text { - Current: } 100 \mathrm{~A}-5000 \text { A optional sensor } \\
\text { - High-speed sampling (max. } 20 \mathrm{MS} / \mathrm{s} \text {; with 54-ch type), Saving data using USB } 2.0 \\
\text { - Measurement of voltage, current, temperature, frequency, distortion, and control } \\
\text { signal(logic). }\end{array}$} \\
\hline & Description & $\begin{array}{l}\text { Multi-Channel Memory } \\
\text { HiCorder ( } 54 \text { channel) }\end{array}$ & \\
\hline & Certification & $\mathrm{CE}$ & \\
\hline & Price (Rp) & 770.500 .000 & \\
\hline
\end{tabular}




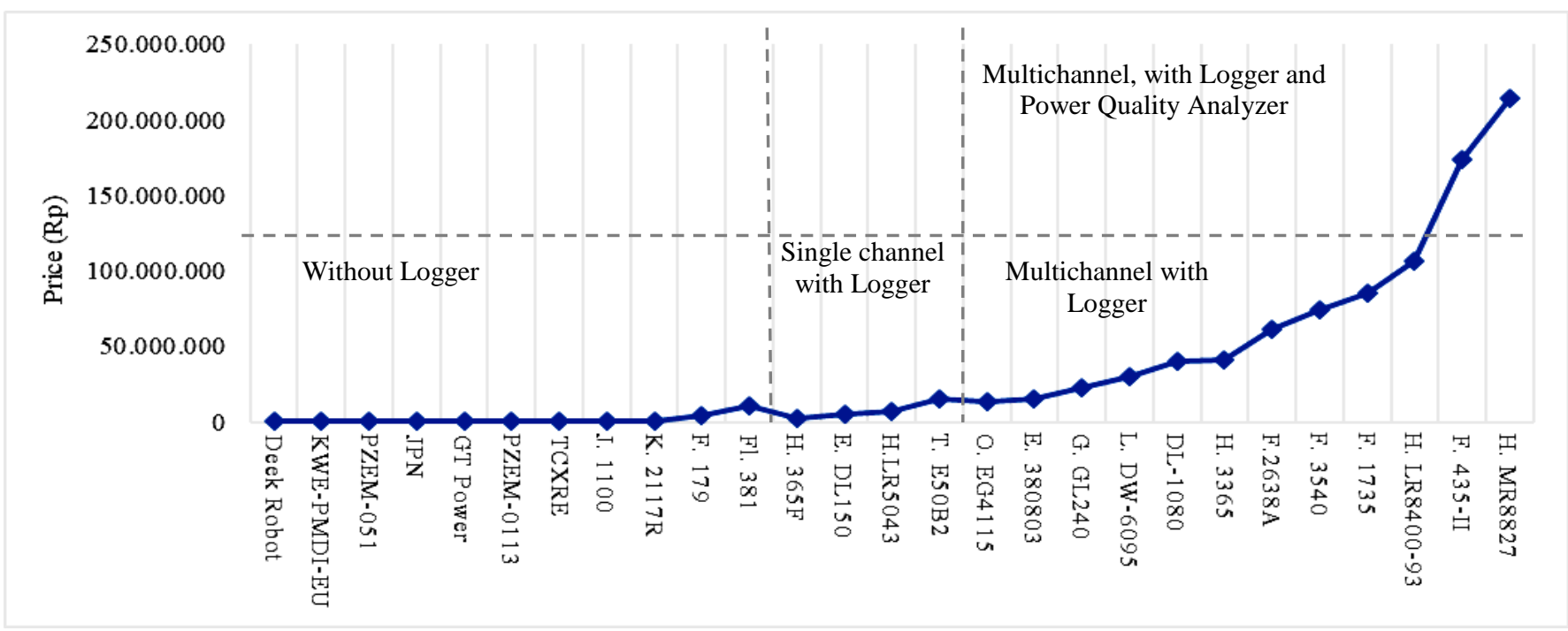

Fig. 2. Price comparison and type of data logger system circulating in the domestic market

Based on Table 6 above and the illustration in Figure 2 , it can be seen that the price of measuring instruments equipped with this data logger system varies from $\mathrm{Rp}$ 2.218.000 to $\mathrm{Rp} 770.500 .000$ depends on several factors, such as how many measurement channels were used by the instruments, measuring range of electrical parameters measured, how many types of measurement parameters can be done by the device, whether or not the device can measure power quality and analyze the condition of the power system, whether or not the device gives a warning if the system condition is interrupted either directly through the alarm or through notification in the application, as well as the display of information that can be displayed on the LCD display, the ability of the device to be interconnected with wireless, as well as the type of storage system used, such as SD cards, USB, or SSD, whether or not integrated with a web server, email, etc. The entire measuring instrument that uses a data logger system has been equipped with labeling from the manufacturer to ensure the quality and safety of the instruments.

The more features possessed by a measuring instrument and data logger system, the more expensive the price of the instrument would be. However, in grouping this instrument it was found that there was no relationship between the number of standardization labels and country of origin of those instruments to its price. The different prices of instruments were caused by many features and the number of channels of the measuring instrument. However, if a device has a standardization label that shows more of the instruments' good quality that would be good because the labeling would guarantee the quality and safety of the instruments that were used.

Some countries strictly regulate the distribution of products in their own countries or imported from other countries through a label/standard, such as the Conformité Européenne (CE) label was applied on products manufactured and sold in European Economic Area (EEA) countries, which includes 28 European Union (EU) countries, Iceland, Norway, Liechtenstein, Turkey and Switzerland; the Regulatory Compliance Mark (RCM) was applied on products marketed in Australia and New Zealand; the Federal Communications
Commission (FCC) was applied on products that marketed in the United States of America (US); the CQC mark was applied on products marketed in China, and the Indonesian National Standard (SNI) was applied on products marketed in Indonesia.

\section{RESEARCH DIRECTION}

To analyze the performance of an electrical system, for example, PLTS, it required a complete data including energy production data every day, environmental monitoring data, such as wind speed data, wind direction, solar radiation data, environmental temperature data, solar module temperature data [6]. To get all the data to analyze the PLTS performance, we need a measurement instrument for monitoring electrical parameters and a data logger system in order to be able to record all the data that has been read by various sensors.

There were various types and prices of electrical parameter measurement instruments, low-cost instruments were not equipped with a data logger system, while expensive ones were already equipped with a data logger system. Data logger systems that were used multiple input channels (Multichannel) to connect information signals from sensors to processors were very expensive compared to data logger systems that were single channel. One example of the single-channel data logger system was the Hantek 365F USB digital data logger recorder multimeter which has a price of $\mathrm{Rp}$ 2.218.000 compared to multichannel data logger systems, such as Hioki MR8740 Multichannel Memory HiCorder (54 channels) this instrument has a price of $\mathrm{Rp}$ 770.500.000. The multichannel data logger has a price up to hundreds of millions of rupiah depending on the number of features and channels that available on the instrument.

In designing a data logger system the main component that must be completed was the microcontroller. Microcontroller consists of components, such as memory, peripherals, and processors. This microcontroller will work like a human brain that will regulate all activities performed by the data logger system, such as the task of regulating the work of sensors 
and sending data read by these sensors into storage media. Nowadays, many types of microcontrollers had been developed and utilized which can be seen in Table 7 .

TABLE VII

TYPE OF MICROCONTROLLERS

\begin{tabular}{|l|l|}
\hline \multicolumn{1}{|c|}{ Name } & \multicolumn{1}{c|}{ Manufacturer } \\
\hline Advanced Virtual RISC (AVR), ATmega & Atmel \\
\hline MSP430x F168 & Texas Instrument \\
\hline 8051 & Intel \\
\hline Peripheral Interface Controller (PIC), PIC 18 & Micro-chip \\
\hline AMR microcontroller & Acorn Computers Ltd \\
\hline
\end{tabular}

In addition to requiring a microcontroller in designing a data logger system also requires a sensor module and other supporting. Like, a temperature sensor module which has functions to temperature measurement, a voltage sensor module which has functions to read the

voltage value, a current sensor module which has functions to read the electric current value, a light intensity sensor module which has functions to measure light intensity with lux units, a pyranometer sensor module which has functions to measure the magnitude of the effect of light radiation on the surface of the field with units $\mathrm{W} / \mathrm{m}^{2}$, wind speed sensor modules to measure wind speed, ethernet module which has functions to connect system to internet to make it easier in data sending, GSM / GPRS module which has functions to transmit data in the form of text messages and call, a Bluetooth module that functions to sending data with Bluetooth media, sd card module that functions to store data in the sd card, a Real-Time Clock (RTC) module that functions for realtime display and recording, LCD module that functions as a local display on the system.

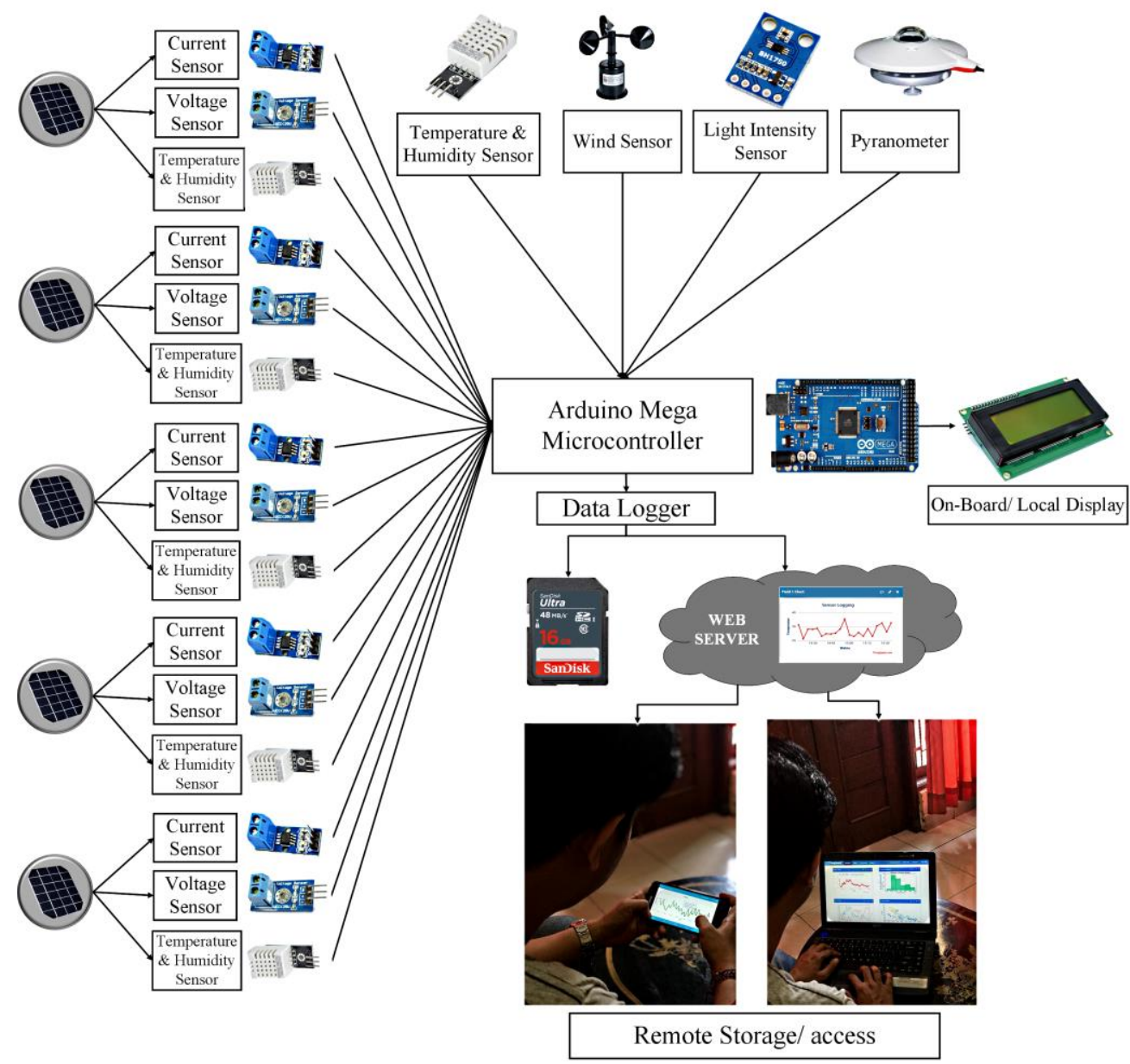

Fig. 3. Low-cost multichannel instrument and IoT

In the future, all monitoring systems must be able to be accessed remotely by utilizing the Wi-Fi network as a link to the remote access/cloud system. Cloud is a digital data storage medium that makes the internet a central server for managing data and also user applications. This system is usually integrated into a concept called the internet of things (IoT). IoT is a technology where an object can send data through a network without requiring human-to-human or human interaction to the computer [7]. The advantage of this IoT technology is that the work done becomes faster, easier, and more efficient. The consequence was important that all monitoring systems use the IoT concept so that measurement data can be accessed remotely by a wireless connection $\mathrm{Wi}-\mathrm{Fi}$.

Because of the importance of data loggers for the operation of electric power systems and also plays an important role in research, it was necessary to design a multichannel data logger system that was capable of measuring electrical parameters and the data was stored in real-time to a cloud system at a low cost. The illustration of a low-cost multichannel data logger system was shown in Figure 3. 


\section{CONCLUSION}

This paper has presented a review of equipment for measuring electrical parameters. The instruments can be divided into two groups, i.e. instrument with or without data logger system. The system without data logger has a lower price compared to the instruments capable of data logging. The instrument without data logger is suitable for indicators of the operation of a machine and its application does not require further analysis. This cost of the equipment varies depending on several factors, such as the measurement range/ limit, the types of electrical parameters that can be measured by the device, AC/DC system, the communication capability/ features of the instruments, as well as information that can be displayed on the LCD display.

Instrument with data logging system have varying prices that depend on several factors, such as the limitation or range of the measurement, types of electrical parameters the instrument can measure, whether or not the device can measure power quality and analyze the conditions of the power system, whether or not the device provides a warning if the system condition is interrupted either directly via an alarm or through notifications, display information that can be displayed on the LCD display, the ability of the device to communicate wirelessly, as well as the type of storage system used such as SD card, USB or SSD, and finally the ability to be integrated with a web server, and email. These instruments normally used for applications that require real-time monitoring, complex systems, applied to vital installations, and systems that require detail analysis. Therefore, using instruments with a data logging tool allows comprehensive monitoring and analysis of the condition of the plant's operation.

The measuring instruments must meet standardization/labeling to ensure the quality of the equipment and safety when used by consumers. Each country has different regulations or policies for the labeling of products sold in the country. Normally, if a measuring instrument does not meet the standard requirements of a country, then the instrument cannot be sold to that country.

\section{REFERENCES}

[1] Fratama, R.A. (2016). Emulasi Monitoring Parameter Kelistrikan Gardu Listrik Di Universitas Muhammadiyah Malang Menggunakan Jaringan Vpn : Server. Other thesis. University Muhammadiyah Malang.

[2] Siswoyo. (2008). Teknik Listrik Industri Jilid 2 untuk SMK. Jakarta, Indonesia: Direktorat Pembinaan Sekolah Menengah Kejuruan, 2-8.

[3] Sanjaya, I. P. G. M., Partha, C. G. I., \& Khrisne, D. C. (2018). Rancang Bangun Sistem Data Logger Berbasis Visual Pada Solar Cell. Majalah Ilmiah Teknologi Elektro, 16(3), 114-121. doi: 10.24843/mite.2017.v16i03p18.

[4] Widodo, MH. S. (2014). Dasar Pengukuran Listrik. Jakarta, Indonesia: Direktorat Pembinaan Sekolah Menengah Kejuruan, 281-284.

[5] Mahzan, N. N., Omar, A. M., \& Mohammad Noor, S. Zaliha. (2015). Design and Development of an Arduino Based Data Logger for Photovoltaic Monitoring System. IJSSST, 17(41), 1-5. DOI: 10.5013/IJSSST.a.17.41.15

[6] Nathawibawa, A. A. N. B. B., Kumara, I. N. S., \& Ariastina, W. G. (2016). Analisis Produksi Energi Dari Inverter pada Gridconnected PLTS 1 MWp di Desa Kayubihi Kabupaten
Bangli. Majalah Ilmiah Teknologi Elektro, 16(1), 131-140. DOI: 10.24843/mite. 1601.18

[7] Yudhanto, Y. (2007, May 15). Apa Itu IoT (Internet of Things) IlmuKomputer. Retrieved Oktober 17, 2019. From: https://ilmukomputer.org.

[8] Shenzhen Deek Technology Co., Ltd. (2013). Deek-Robot BL-02 Digital Voltage Current Power Meter adjustable voltmeter ammeter Wattmeter Power: Instruction manual. China: DeekRobot

[9] Sandal plc. (2018). Energy Power Analyzer KWE-PMDI-EU Digital Watt Voltage Watt Meter Ampere: User Guide. England: Energenie.

[10] Ningbo Peacefair Electronic Technology Co., Ltd. (2017).PZEM051 DC 100V 100A 4 in 1 Voltmeter Amperemeter Wattmeter Energy meter: Product Catalog. China: Peacefair.

[11] Sinaga, S. (2018). JPN DC Watt Ampere Volt. Retrieved Oktober 18, 2019. From: https://www.bukalapak.com/

[12] Shenzhen G.T.Hobbies.Co., Ltd. (2009). GT Power 150A RC High Precision Power Analyzer \& Watt Meter: Instruction manual. China: G.T Power RC.

[13] Ningbo Peacefair Electronic Technology Co., Ltd. (2017). PZEM-013 DC LCD Display Digital Current Voltage Solar Power Meter Multimeter Ammeter Voltmeter: Product Catalog. China: Peacefair.

[14] Mizhar. (2018). TCXRE AC 80-300 V LCD 100A Volt Watt Power Meter Pengukur Amper Pengukur Tegangan. Retrieved Oktober 18, 2019. From: https://www.tokopedia.com.

[15] Hangzhou Juntek Instrumentation Co., Ltd. (2018). JUNTEK DC VAT-1100 Multifunctional Wireless Digital Bi-directional Voltage Current Power Meter Ammeter Voltmeter Capacity Coulomb Counter: Instruction manual. China: JUNTEK.

[16] Kyoritsu Electrical Instruments Works, Ltd. (2015). AC Digital Clamp Meter KEW 2117R: Instruction manual. Japan: KYORITSU.

[17] Fluke Corporation. (2003). Fluke 179 True RMS Digital Multitester Multitesters: Instruction manual. Washington: Fluke.

[18] Fluke Corporation. (2010). 381 Remote Display True-RMS AC/DC Clamp Meter with iFlex ${ }^{\mathrm{TM}:}$ Instruction manual. Washington: Fluke.

[19] Qingdao Hantek Electronic Co., Ltd. (2006). Hantek 365F USB digital data logger recorder multimeter voltage and current Instruction manual. China: Hantek.

[20] Extech Instruments Corporation. (2013). True RMS AC Voltage/Current Datalogger DL 150: Instruction manual. Nashua: Extech

[21] Hioki E.E. Corporation. (2013). Hioki LR5043-20 Single Channel Voltage Logger: Instruction manual. Japan: Hioki.

[22] Onset Computer Corporation. (2018). EG4115 Core - 15 Input Meter Data Logger: Instruction manual. United States: Onset.

[23] Onset Computer Corporation. (2013). T-VER-E50B2 Compact Power and Energy Meter: Instruction manual. United States: Onset.

[24] Extech Instruments Corporation. (2010). Extech 380803 True RMS Power Analyzer/Appliance Tester and Datalogger: Instruction manual. Nashua: Extech.

[25] GRAPHTEC Corporation. (2018). Graphtec GL240 10 channel analog: Instruction manual. Japan: Graphtec.

[26] Lutron Electronics Co., Inc. (2016). Lutron DW-6095 power analyzer real-time data logger: Instruction manual. New York: Lutron.

[27] Chauvin Arnoux, Inc. d.b.a. AEMC Instruments. (2019). AEMC DL-1080 Data Logger: Instruction manual. United State: AEMC.

[28] Hioki E.E. Corporation. (2018). Hioki PW 3365-20 Clamp Power Logger: Instruction manual. Japan: Hioki.

[29] Fluke Corporation. (2016). Fluke 2638A/05 Hydra Series III 22 Channel Data Acquisition System: Instruction manual. Washington: Fluke.

[30] Fluke Corporation. (2016). Fluke 3540 FC KIT Three-Phase Power Monitor: Instruction manual. Washington: Fluke

[31] Fluke Corporation. (2006). Fluke 1735 Three-Phase Power Logger: Instruction manual. Washington: Fluke.

[32] Hioki E.E. Corporation. (2015). Hioki LR8400-93 PV Power Verifier: Instruction manual. Japan: Hioki.

[33] Fluke Corporation. (2006). Fluke 435-II Three-Phase Power Quality And Energy Analyzer: Instruction manual. Washington: Fluke. 
[34] Hioki E.E. Corporation. (2015). Memory Hicorder MR8827: Instruction manual. Japan: Hioki.

[35] Hioki E.E. Corporation. (2015). Memory Hicorder MR8740: Instruction manual. Japan: Hioki.

[36] Hanson, D. (2005). Ce marking, product standards, and world trade. Retrieved November 27, 2019. from https://books.google.co.id/.

[37] Medi, N. (2019, June 28). What are Electronic Products Certifications? - Meee Services. Retrieved October 12, 2019, from https://www.meee-services.com/electronic-productcertifications/.

[38] RoHS Guide. (n.d.). RoHS 10 Restricted Substances. Retrieved November 24, 2019, from https://www.rohsguide.com.

[39] BSN Badan Standardisasi. (2017, December 11). Tentang SNI. Retrieved November 2, 2019, from http://bsn.go.id.

[40] BSN Badan Standardisasi Nasional. (2018, December 5). Pemberlakuan Wajib SNI Peralatan Listrik, Lindungi Konsumen. Retrieved December 18, 2019, from http://www.bsn.go.id.

[41] Humas BSN (2018, Desember 5). Pemberlakuan Wajib SNI Peralatan Listrik, Lindungi Konsumen [Online]. Available www.bsn.go.id

[42] Robinson, P. W. (2016). Regulatory compliance mark: For Australia/New Zealand electrical and electronic equipment. 2016 IEEE Symposium on Product Compliance Engineering (ISPCE), 1-27. DOI: 10.1109/ispce.2016.7492843

[43] Rheinland, T. Ü. V. (n.d.). Electric and Electronic Components. Retrieved November 1, 2019, from https://www.tuv.com. 